\title{
Relation between medial temporal atrophy and functional brain activity during memory processing in Alzheimer's disease: a combined MRI and SPECT study
}

\author{
G E J Garrido, S S Furuie, C A Buchpiguel, C M C Bottino, O P Almeida, C G Cid, \\ C H P Camargo, C C Castro, M F Glabus, G F Busatto
}

See editorial commentary, this issue pages $470-1$.

J Neurol Neurosurg Psychiatry 2002;73:508-516

See end of article for authors' affiliations

Correspondence to:

Dr G E J Garrido, Centro

de Medicina Nuclear-USP,

Rua Dr Ovidio Pires de

Campos s/n, 05403-010,

São Paulo-SP, Brazil;

neuroimage@hcnet.usp.br

Received

4 January 2002

In revised form

18 June 2002

Accepted 3 July 2002
Objective: To investigate the relation between atrophy of the hippocampal region and brain functional patterns during episodic memory processing in Alzheimer's disease.

Patients and methods: Whole brain structural magnetic resonance imaging (MRI) data and single photon emission computed tomography (SPECT) measures of regional cerebral blood flow ( $\mathrm{rCBF}$ ) were obtained during a verbal recognition memory task in nine subjects with mild Alzheimer's disease and 10 elderly healthy controls. Using the statistical parametric mapping approach, voxel based comparisons were made on the MRI data to identify clusters of significantly reduced grey matter concentrations in the hippocampal region in the Alzheimer patients relative to the controls. The mean grey matter density in the voxel cluster of greatest hippocampal atrophy was extracted for each Alzheimer subject. This measure was used to investigate, on a voxel by voxel basis, the presence of significant correlations between the degree of hippocampal atrophy and the rCBF SPECT measures obtained during the memory task.

Results: Direct correlations were detected between the hippocampal grey matter density and rCBF values in voxel clusters located bilaterally in the temporal neocortex, in the left medial temporal region, and in the left posterior cingulate cortex during the memory task in the Alzheimer's disease group $(p<0.001)$. Conversely, measures of hippocampal atrophy were negatively correlated with $\mathrm{rCBF}$ values in voxel clusters located in the frontal lobes, involving the right and left inferior frontal gyri and the insula $(p<0.001)$.

Conclusions: Hippocampal atrophic changes in Alzheimer's disease are associated with reduced functional activity in limbic and associative temporal regions during episodic memory processing, but with increased activity in frontal areas, possibly on a compensatory basis.
T he cardinal clinical feature of Alzheimer's disease is a pronounced loss of episodic memory. The disease involves an uneven and progressive loss of neurones, associated with the development of amyloid containing neuritic plaques and neurofibrillary tangles. These pathological changes occur first and with greatest severity in medial temporal structures, including the hippocampus and parahippocampal gyrus ${ }^{23}-$ regions that are thought to be critical for episodic memory processes. ${ }^{4}$ The prominence of medial temporal abnormalities in early Alzheimer's disease has been confirmed by many in vivo magnetic resonance imaging (MRI) studies, which have reported significant grey matter atrophy in the hippocampal region in Alzheimer subjects relative to healthy controls, ${ }^{5-9}$ often in direct proportion to the degree of memory impairment. ${ }^{6}$

Functional imaging techniques have also been used to compare groups of patients with Alzheimer's disease with healthy controls, most often measuring resting regional cerebral blood flow (rCBF) or regional glucose metabolism with positron emission tomography (PET) or single photon emission computed tomography (SPECT). ${ }^{11-14}$ A pattern of hypoactivity in the temporal and parietal association cortices in Alzheimer patients is the most characteristic finding in those studies, detected either by visual inspection or by conventional quantitative analyses using regions of interest (ROI). More recent studies, involving equipment of higher spatial resolution and voxel based statistical parametric mapping (SPM) methods for image analysis, have also been able to document resting activity decrements in the medial temporal region in Alzheimer's disease, as well as in the posterior cingulate gyrus and precuneus in very early stages of the disorder. ${ }^{15-17}$

Several functional imaging studies have also examined brain activity patterns in Alzheimer patients and healthy controls during the performance of memory activation paradigms, using PET, SPECT, or functional MRI. ${ }^{18-24}$ These studies have often employed tasks involving episodic memory retrieval processes, which in healthy subjects engage a distributed network of regions including the medial temporal, prefrontal, and lateral parietal cortices, as well as the precuneus, cingulate gyrus, and cerebellum. ${ }^{25}$ In Alzheimer patients, activation of the medial temporal region during memory tasks is infrequent. ${ }^{192}$ On the other hand, patients with mild Alzheimer's disease often show memory related activity increases in the prefrontal cortex, with a broader spatial extent relative to healthy controls. ${ }^{19223}$ These frontal activity increases have often been interpreted as a functional compensatory response, related to the increased effort needed by patients with Alzheimer's disease to perform the tasks. ${ }^{196}$

The relation between findings from the structural and functional imaging fields in Alzheimer's disease has been little

Abbreviations: CAMCOG, CAMDEX section for assessment of cognitive function; CAMDEX, Cambridge mental disorders of the elderly examination; $\mathrm{PET}$, positron emission tomography; $\mathrm{rCBF}$, regional cerebral blood flow; SPECT, single photon emission computed tomography; SPM, statistical parametric mapping; VBM, voxel based morphometry 
Table 1 Clinical and neuropsychological characteristics of subjects with Alzheimer's disease $(n=9)$ and healthy controls $(n=10)$

\begin{tabular}{|c|c|c|c|c|}
\hline & Alzheimer's disease & Healthy controls & Statistical test & Significance \\
\hline \multicolumn{5}{|l|}{ Clinical variables } \\
\hline $\operatorname{Sex}(M: F)$ & $7: 2$ & $6: 4$ & $\chi^{2}=0.69$ & $p=0.405$ \\
\hline Age (years) (mean (SD), range) & $70(7.75), 62$ to 82 & $70.10(5.65), 64$ to 79 & $t=-0.03$ & $p=0.975$ \\
\hline Handedness (right:non-right) & $9: 0$ & $9: 1$ & $\chi^{2}=0.95$ & $p=0.330$ \\
\hline Years of education (mean (SD)) & $10.67(3.08)$ & $6.90(4.63)$ & $t=2.06$ & $\mathrm{p}=0.055$ \\
\hline Social class $(A / B / C / D / E)$ & $0 / 6 / 3 / 0 / 0$ & $0 / 2 / 5 / 3 / 0$ & $\chi^{2}=5.46$ & $p=0.065$ \\
\hline MMSE (mean (SD), range) & $21.78(1.86), 18$ to 25 & $29.10(0.57), 28$ to 30 & $t=-11.91$ & $p<0.001$ \\
\hline MADRS (mean (SD), range) & $13.11(5.67), 4$ to 22 & $2.60(2.99), 0$ to 8 & $t=5.14$ & $p<0.001$ \\
\hline \multicolumn{5}{|l|}{ Scores on neuropsychological tests } \\
\hline \multicolumn{5}{|l|}{ CAMCOG (mean (SD)) } \\
\hline Language comprehension & $8.11(0.93)$ & $8.80(0.63)$ & $t=-1.91$ & $p=0.073$ \\
\hline Praxis & $9.89(1.17)$ & $11.00(1.25)$ & $t=-1.99$ & $p=0.062$ \\
\hline Remote memory & $3.22(1.64)$ & $5.00(0.94)$ & $t=-2.93$ & $p=0.009$ \\
\hline Recent memory & $1.44(1.24)$ & $3.50(0.71)$ & $t=-4.51$ & $p<0.001$ \\
\hline Category fluency (mean (SD)) & $10.33(3.00)$ & $13.90(3.7)$ & $t=-2.29$ & $p=0.035$ \\
\hline FOME delayed recall (mean (SD)) & 4.89 (3.89) & $9.75(0.46)$ & $t=-3.50$ & $p=0.003$ \\
\hline
\end{tabular}

explored to date. There are reports of significant correlations between atrophy in the hippocampal region, as assessed by computed tomography (CT) or MRI, and reduced resting temporal-parietal metabolism in Alzheimer patients. ${ }^{27-29}$ These findings are thought to indicate the remote effects of hippocampal structural lesions on the function of connected neocortical sites. ${ }^{29}$ However, no imaging study to date has directly investigated the relation between brain atrophy and function during the performance of memory activation paradigms. Thus it is difficult to ascertain how the primary structural abnormalities in the hippocampal region might influence the overall brain activity patterns seen in Alzheimer patients during memory tasks.

In the present investigation, we have studied a sample of patients with mild Alzheimer's disease and elderly healthy controls using both structural MRI, to measure hippocampal atrophy, and the SPECT technique, to measure rCBF patterns during an episodic memory retrieval task. The SPM approach was applied to the data obtained with both imaging modes, in order to investigate-on a voxel by voxel basis-the presence of significant correlations between the degree of hippocampal atrophy and rCBF values obtained during the memory task. We aimed to investigate the patterns of correlation between hippocampal grey matter changes and rCBF values in cortical regions where hypofunctional patterns could be predicted a priori in Alzheimer patients relative to controls, including the temporal and parietal association cortices, the medial temporal region, the cingulate cortex, and the precuneus. In addition, we wished to ascertain whether significant correlations would also be present between hippocampal grey matter changes and rCBF values in the prefrontal cortex, where activity increases were predicted to occur during the memory task.

\section{METHODS}

\section{Subjects and clinical assessment}

Nine patients with Alzheimer's disease meeting NINDS/ ADRDA criteria for probable Alzheimer's disease ${ }^{30}$ took part in the study. They were interviewed with the Cambridge mental disorders of the elderly examination ${ }^{31}$ (CAMDEX), and examined with cranial CT, urinalysis, and blood tests including full blood count, liver, renal, and thyroid function tests, vitamin B-12 and folate levels, and syphilis serology. Patients with a Hachinski ischaemic score of $\geqslant 4$ or a history of significant hearing deficits were excluded. All patients had scores on the mini-mental state examination (MMSE) $)^{32}$ of $\geqslant 18$.
Three Alzheimer patients were not on any psychotropic drugs; the remaining six were receiving acetylcholinesterase inhibitor drugs (five patients), antidepressants (two patients), and antipsychotic drugs (one patient).

A control group of 10 healthy elderly subjects was also studied. They were free of symptoms suggestive of physical or mental disorder, based on the CAMDEX interview, general medical questioning, and physical and neurological examination. All healthy controls had an MMSE score of $\geqslant 28$, and a Hachinski ischaemic score lower than 4. No subject in this group was taking any class of psychotropic drug.

A summary of the demographic and clinical characteristics of the two groups is given in table 1. The Alzheimer's disease and healthy control groups did not differ significantly in terms of age, sex, or handedness. There were trends toward a greater number of years of education and a higher socioeconomic status as assessed by standardised criteria ${ }^{33}$ in the Alzheimer group (table 1).

Subjects from both groups also underwent a neuropsychological test battery including subitems for language comprehension, praxis, remote memory, and recent memory from the CAMDEX section for assessment of cognitive function ${ }^{31}$ (CAMCOG); a category verbal fluency task ${ }^{34}$; and the Fuld object-memory evaluation (FOME). ${ }^{35}$ Mean scores on these neuropsychological variables for Alzheimer's disease patients and controls are also given in table 1 .

\section{MRI acquisition and analysis}

MRI images were obtained using a 1.5 Tesla Philips Gyroscan S15-ACS scanner (Philips Medical Systems, Eindhoven, Netherlands). A series of contiguous $1.2 \mathrm{~mm}$ thick coronal images across the entire brain was acquired, using a Tl weighted fast field echo sequence (time of echo (TE) $=9 \mathrm{~ms}$, time of repetition $(\mathrm{TR})=30 \mathrm{~ms}$, flip angle $=30^{\circ}$, field of view $=240 \mathrm{~mm}$, $205 \times 256$ matrix) .

Images were analysed with statistical parametric mapping software (SPM99), using the voxel based morphometric (VBM) approach as described by Ashburner and Friston. ${ }^{36}$ Images were spatially normalised with linear and non-linear transformations into a stereotaxic space that approximates the space defined in the atlas of Talairach and Tornoux, ${ }^{37}$ with a final voxel size of $[2 \mathrm{~mm}]^{3}$. The normalised images were then segmented into grey matter, white matter, and cerebrospinal fluid by using a modified mixture model cluster analysis technique. ${ }^{36}$ This method uses prior probability maps which are overlaid onto the images in order to classify each voxel in terms of its probability of belonging to a particular tissue class. 
The final values for the belonging probabilities are in the range of 0 to 1 , with most voxels classified close to one of the two extremes. Finally, in order to reduce variation caused by individual differences in sulcal and gyral anatomy, the grey matter segments were smoothed using an isotropic Gaussian kernel of $8 \mathrm{~mm}$ full width at half maximum.

The mean grey matter concentration in Alzheimer patients and healthy controls was compared at all brain voxels which had values above an absolute grey matter threshold of 0.05 (resulting in a search volume of approximately 250000 voxels). The statistics for each voxel were transformed to $\mathrm{Z}$ scores and displayed as a statistical parametric map (SPM) into standard space, at a threshold probability of $\mathrm{Z}=2.33$ $(p<0.01)$. The resulting SPM was inspected for the presence of any clusters of reduced medial temporal grey matter concentration in Alzheimer patients relative to controls, in which the voxel of peak statistical difference attained a probability ( $p$ ) value of $<0.001(\mathrm{Z}>3.09)$.

After the cluster of greatest medial temporal grey matter atrophy in Alzheimer patients was identified, a Matlab program was used to obtain the coordinates of all voxels included in this cluster that surpassed the initial cut off of $\mathrm{Z}>2.33$. The mean intensity values for this cluster were then calculated for all Alzheimer subjects using the smoothed MR images, providing a measure of the mean grey matter concentration within that region.

The use of the liberal cut off of $\mathrm{Z}=2.33$ was intended to favour the inclusion of a greater number of voxels in the selected medial temporal cluster, in order to provide a measure that would be representative of the degree of grey matter atrophy over a large extension of hippocampal tissue. The mean intensity values for each Alzheimer subject in this cluster were stored for subsequent use in the correlational analysis with the rCBF SPECT data.

\section{SPECT image acquisition}

The SPECT acquisition was conducted in all subjects within two weeks of the MRI session. A split dose ${ }^{99 m}$ Tc-HMPAO technique was used, allowing the acquisition of two rCBF SPECT scans within the same session, each after the performance of a different cognitive task. ${ }^{38}$

Initially, subjects were kept in a quiet testing room, where a venous cannula was inserted in the right arm. They were positioned supine with eyes closed, wearing earphones through which they received the instructions for the first (control) task. During this task, subjects heard the words "yes" (24 times) and "no" (36 times) randomly alternated, at a rate of one word every five seconds. Subjects were instructed to press a button with their right hand every time they heard the word "yes". After one minute into this task, a first $15 \mathrm{mCi}{ }^{99 \mathrm{~m}} \mathrm{Tc}$-HMPAO injection was given. The task was continued for an additional period of four minutes, after which the subjects were taken to the scanning room for the first rCBF SPECT acquisition.

After the first SPECT scan, subjects returned to the testing room for the performance of the verbal memory task. During an initial learning stage of the memory experiment, subjects were presented, through their earphones, with a list of 12 common Portuguese words (at a rate of one word every two seconds), and they attempted to memorise these words over five trials. After an interval of 90 seconds, the recognition phase of the experiment was started. Subjects were presented with a list of 60 words (at a rate of one word every five seconds), including the 12 words previously learned (presented two times each), randomly mixed with 36 new words. Subjects were instructed to press the button each time they heard a word that had been presented during the learning phase of the experiment. The second $15 \mathrm{mCi}{ }^{99 \mathrm{~m}} \mathrm{Tc}-\mathrm{HMPAO}$ injection was given during the recognition memory task. The task was continued for another four minutes, after which the second SPECT scan was acquired.
For both the baseline and recognition memory tasks, visual analogue scales $(0-10)$ were used to document self reported anxiety; measures were taken five minutes after tracer injection, when subjects were asked to rate how they felt during the performance of the task they had just completed.

SPECT images were acquired with a double detector GE-OPTIMA system (General Electric Medical Systems, Milwaukee, Wisconsin, USA). For both the baseline and memory scans, high resolution collimators were used, with 128 views acquired on a $64 \times 64$ matrix ( 10 seconds per view). Images were reconstructed as cubic voxels $\left([4.5 \mathrm{~mm}]^{3}\right)$ by filtered back projection (ramp filter), with Butterworth postfiltering (cut off $=0.59$, order number $=10$ ). Attenuation correction was performed using the algorithm of Chang (uniform linear attenuation coefficient $=0.12 \mathrm{~cm}^{-1}$ ).

\section{SPECT image analysis}

The rCBF SPECT images were also analysed using SPM99. Initially, the pair of baseline and memory scans of each subject were realigned (sinc interpolation) with each other, and spatially normalised to standard anatomical space with a final voxel size of $[2 \mathrm{~mm}]^{3}$. Given the stability of the ${ }^{99 m}$ Tc-HMPAO distribution in the brain for several hours after injection, ${ }^{39}$ the regional count density measured during the memory SPECT scan represented a superimposition of the tracer uptake patterns obtained during the second injection over the uptake patterns corresponding to the first, baseline injection. Therefore, in order to obtain a net measure of the ${ }^{99 m} \mathrm{Tc}-$ HMPAO uptake originating from the second injection, the decay corrected, normalised baseline scan was subtracted from the normalised memory scan on a voxel by voxel basis. The normalised baseline and subtracted memory rCBF SPECT scans of each subject were then both smoothed with an isotropic $12 \mathrm{~mm}$ (FWHM) Gaussian kernel.

First, the mean regional ${ }^{99 m}$ Tc-HMPAO uptake patterns obtained during the memory task were statistically compared between Alzheimer patients and controls for each voxel of the smoothed brain volume using unpaired $t$ tests. Only voxels with signal intensities above a threshold of 0.5 relative to the mean global were entered in each analysis (resulting in a search volume of approximately 272900 voxels). In order to account for interindividual differences in global cerebral blood flow, the regional ${ }^{99 \mathrm{~m}}$ Tc-HMPAO uptake was standardised to the mean global uptake using proportional scaling. To account for the influence of memory task performance on the rCBF patterns elicited in Alzheimer subjects and controls, the between-group comparisons were repeated with covariance for scores on the word recognition test.

Second, a $2 \times 2$ factorial analysis was undertaken in order to compare the patterns of rCBF activation during the memory task relative to the control condition between Alzheimer patients and controls. The condition and subject effects were estimated according to the general linear model at each voxel, ${ }^{40}$ and linear contrasts were used to identify significant differences between conditions and subjects.

Finally, voxel based correlational analyses were performed between $\mathrm{rCBF}$ values in the Alzheimer's disease group and the mean values of grey matter concentration in the medial temporal region extracted from the VBM analysis described above. In order to account for the confounding effect of intersubject differences in global grey matter atrophy, this correlational analysis included a measure of the total amount of grey matter in each subject (given by the sum of the intensity values of all voxels from the smoothed grey matter MR images) as a confounding covariate.

In all analyses above, resulting statistics were transformed to $\mathrm{Z}$ scores, thresholded at $\mathrm{Z}=2.33$, and displayed as SPMs. The SPMs generated from the correlational analyses between medial temporal atrophy and $\mathrm{rCBF}$ values were first inspected for the presence of significant findings $(p<0.001, \mathrm{Z}>3.09)$ in 
L
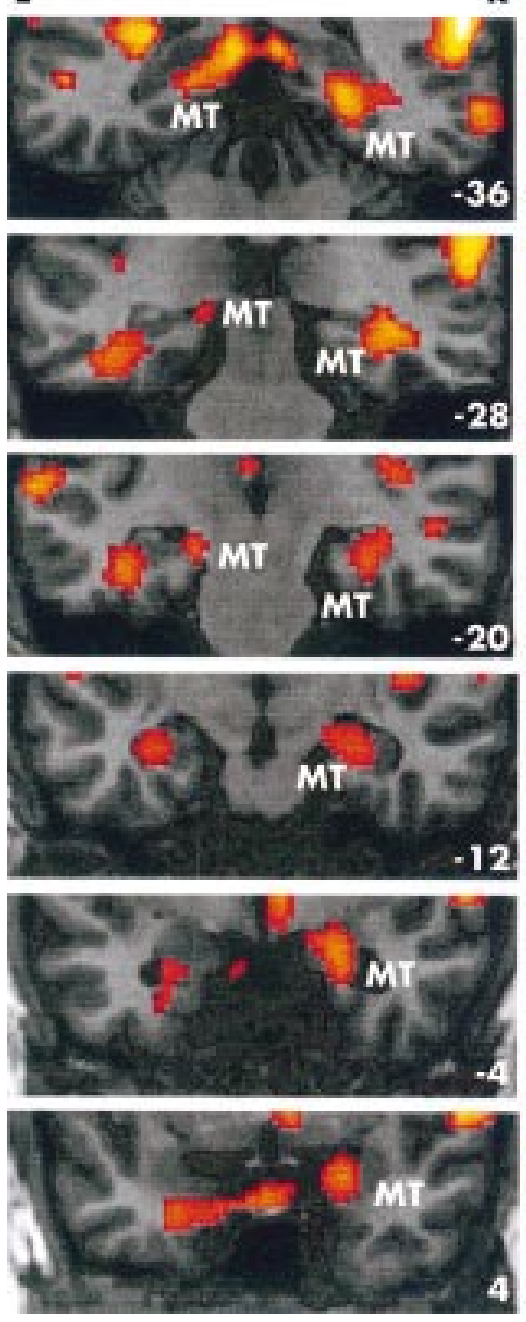

$\mathbf{R}$
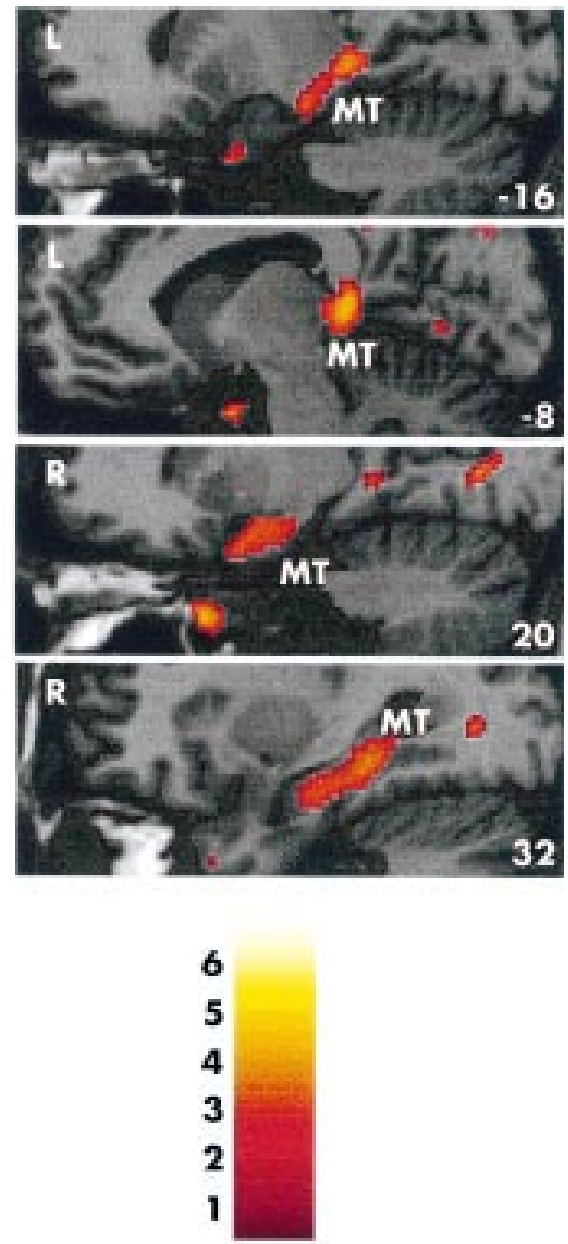

Figure 1 Clusters of reduced grey matter concentration in the medial temporal region in patients with Alzheimer's disease ( $\mathrm{n}=9$ ) relative to healthy controls $(n=10$ ) are shown in red and yellow, at the statistical threshold of $Z=2.33$ ( $<<0.01$ ), overlaid on coronal sections (left column) and sagittal sections (right column) from a reference brain spatially normalised according to the Tailarach and Tornoux stereotactic atlas. ${ }^{37}$ The numbers at the right bottom corner provide the $y$ and $x$ axis coordinate values of each coronal and sagittal view, respectively. The areas with the MT label correspond to the bilateral cluster of greatest grey matter reduction in Alzheimer patients, which had its two voxels of peak statistical significance located, respectively, in the medial border of the left posterior parahippocampal gyrus $\left(Z_{\max }=3.35 ; p<0.0005\right.$; $x, y, z=-6,-38,6)$, and in the right posterior parahippocampal gyrus $\left(Z_{\max }=3.21 ; p<0.001 ; x, y, z=26,-40,-6\right)$. This cluster, which included a total of 1162 voxels, is shown in the figure extending anteriorly on both hemispheres, to reach the enthorinal cortex and the amygdala on the right side and the mid portion of the hippocampus and parahippocampal gyrus on the left side. The mean grey matter concentration in this hippocampal cluster was extracted for all Alzheimer patients and used for the subsequent correlational analysis with the rCBF measures obtained during cognitive activation. L, left hemisphere; R, right hemisphere.

the regions where hypofunctional patterns could be predicted a priori in Alzheimer patients relative to controls-namely the temporal and parietal association cortices, the medial temporal region, the cingulate cortex, and the precuneus. Second, the SPMs were inspected for significant correlations $(p<0.001)$ involving the frontal lobes, where rCBF increases could be predicted to occur during the memory task. Finally, the SPMs were inspected for the presence of significant correlations between medial temporal atrophy and rCBF values in unpredicted regions; the latter correlations were reported as significant only if resisting correction for multiple comparisons based on Gaussian random field theory $(\mathrm{p}<0.05){ }^{41}$

\section{RESULTS}

\section{Between-group structural MRI comparisons}

The VBM analysis showed widespread clusters of significantly reduced grey matter concentration in Alzheimer patients relative to the controls at the $\mathrm{p}<0.001$ level, located mainly in the temporal and parietal lobes. As predicted, one of these clusters was located in the medial temporal region, encompassing the hippocampus/parahippocampal gyrus on both hemispheres (fig 1). This cluster contained 1162 voxels that surpassed the initial threshold of $Z=2.33$, and had its two peaks of statistical difference located, respectively, in the medial border of the left posterior parahippocampal gyrus $\left(\mathrm{Z}_{\max }=3.35 ; \mathrm{p}<0.0005\right.$; $\mathrm{x}, \mathrm{y}, \mathrm{z}=-6,-38,6)$, and in the right posterior parahippocampal gyrus $\left(Z_{\max }=3.21 ; p<0.001 ; x, y, z=26,-40,-6\right)$. The cluster extended anteriorly on both hemispheres along the long axis of the hippocampus/parahippocampal gyrus, reaching the enthorinal cortex and the amygdala on the right side, and the mid portion of the hippocampus on the left side (fig l). The mean grey matter concentration in this medial temporal focus was extracted for each Alzheimer subject and used for the subsequent correlational analysis with rCBF data reported below.

When the between-group VBM comparisons of grey matter concentration were repeated after controlling for global grey matter differences, the above cluster of reduced hippocampal 
grey matter concentration in Alzheimer patients did not retain statistical significance at the $p<0.001$ level; this suggested that the degree of grey matter decrement in this region did not surpass the level of overall brain atrophy in the Alzheimer group. The detailed VBM results for the other brain regions have been described in a separate report, including an extended sample of Alzheimer patients. ${ }^{42}$

\section{Behavioural results during the rCBF measurements}

All subjects in both groups presented a 100\% rate of correct responses during the "yes-no" control condition with the exception of two Alzheimer patients and one healthy control, who missed one "yes" hit each. All subjects in the Alzheimer group were also able to comply with the memory activation paradigm, but presented a trend towards a lower mean (SD) number of correct word recognition scores when compared with healthy controls (19.2 (2.9) $v 21.1 \quad(3.1), t=1.60$, $\mathrm{p}=0.122)$, as well as a significantly greater mean number of false positive hits (4.8 (5.7) $v 0.8(0.9), t=2.58, \mathrm{p}=0.016)$. Also, the mean number of words recalled during the learning phase of the experiment before the SPECT procedure was lower in the Alzheimer patients relative to the controls (5.4 (1.6) $v 6.9(0.9), t=3.09, \mathrm{p}=0.005)$.

\section{Comparisons of rCBF patterns between Alzheimer patients and healthy controls}

The between-group SPM comparisons of SPECT data showed no significant rCBF increases in the Alzheimer group relative to the controls during the memory task. On the other hand, clusters of reduced rCBF in Alzheimer patients relative to controls ( $p<0.001$, uncorrected for multiple comparisons) were detected in temporal and parietal regions, as shown in fig 2. These clusters were located in: the right anterior superior temporal gyrus Brodmann area (BA 22) $\quad\left(\mathrm{Z}_{\max }=4.06\right.$; $\mathrm{x}, \mathrm{y}, \mathrm{Z}=64,-2,4)$; the right and left precuneus (BA7) $\left(\mathrm{Z}_{\max }=3.46 ; \mathrm{x}, \mathrm{y}, \mathrm{z}=6,-76,44\right)$, extending anteriorly towards the posterior cingulate gyrus (BA31); the left hippocampus $\left(Z_{\max }=3.09 ; x, y, z=-30,-20,-10\right)$; and the right postcentral gyrus $\left(Z_{\max }=3.57 ; x, y, z=54,-24,58\right)$. Additional foci of reduced $\mathrm{rCBF}$ in the Alzheimer group $(\mathrm{p}<0.001$, uncorrected) were detected in the upper portion of the left superior frontal gyrus (BA6) $\left(\mathrm{Z}_{\max }=3.25 ; \mathrm{x}, \mathrm{y}, \mathrm{z}=-16,-4,76\right)$, and in the anterior portion of the right medial frontal gyrus (BA10) $\left(Z_{\max }=3.77 ; x, y, z=6,68,-14\right)$ (fig 2). None of those regions showed significantly decreased rCBF values in Alzheimer patients compared with controls when the SPECT scans obtained during the control condition were compared between the two groups.

When the between-group comparisons of rCBF patterns during the word recognition task were repeated with covariance for memory performance, significant rCBF reductions in Alzheimer patients relative to controls were detected in similar regions as reported above ( $\mathrm{p}<0.001$, uncorrected). These included: the right anterior superior temporal gyrus (BA22) $\quad\left(Z_{\max }=3.60 ; x, y, z=64,-2,4\right)$; the right and left precuneus (BA7) $\left(Z_{\max }=3.22 ; x, y, z=8,-76,46\right)$; the right postcentral gyrus $\left(Z_{\max }=3.28 ; x, y, z=52,-24,58\right)$; the upper portion of the left superior frontal gyrus (BA6) $\left(Z_{\max }=3.26\right.$; $\mathrm{x}, \mathrm{y}, \mathrm{Z}=-12,22,60)$; and the anterior portion of the right medial frontal gyrus (BA10) $\left(\mathrm{Z}_{\max }=3.37 ; \mathrm{x}, \mathrm{y}, \mathrm{z}=6,66,-16\right)$. In addition, a focus of reduced $\mathrm{rCBF}$ in Alzheimer patients emerged in the left lateral parietal cortex (inferior parietal lobule; BA40) $\left(Z_{\max }=3.77 ; x, y, z=-48,-42,58\right)$. On the other hand, the above focus of reduced left hippocampal rCBF that had been detected in the Alzheimer patients failed to reach statistical significance after covariance for recognition memory scores $\left(Z_{\max }=2.67\right)$.

Factorial analysis investigating between-group differences in the activation patterns elicited by the memory task revealed greater $\mathrm{rCBF}$ increases in the control group relative to the

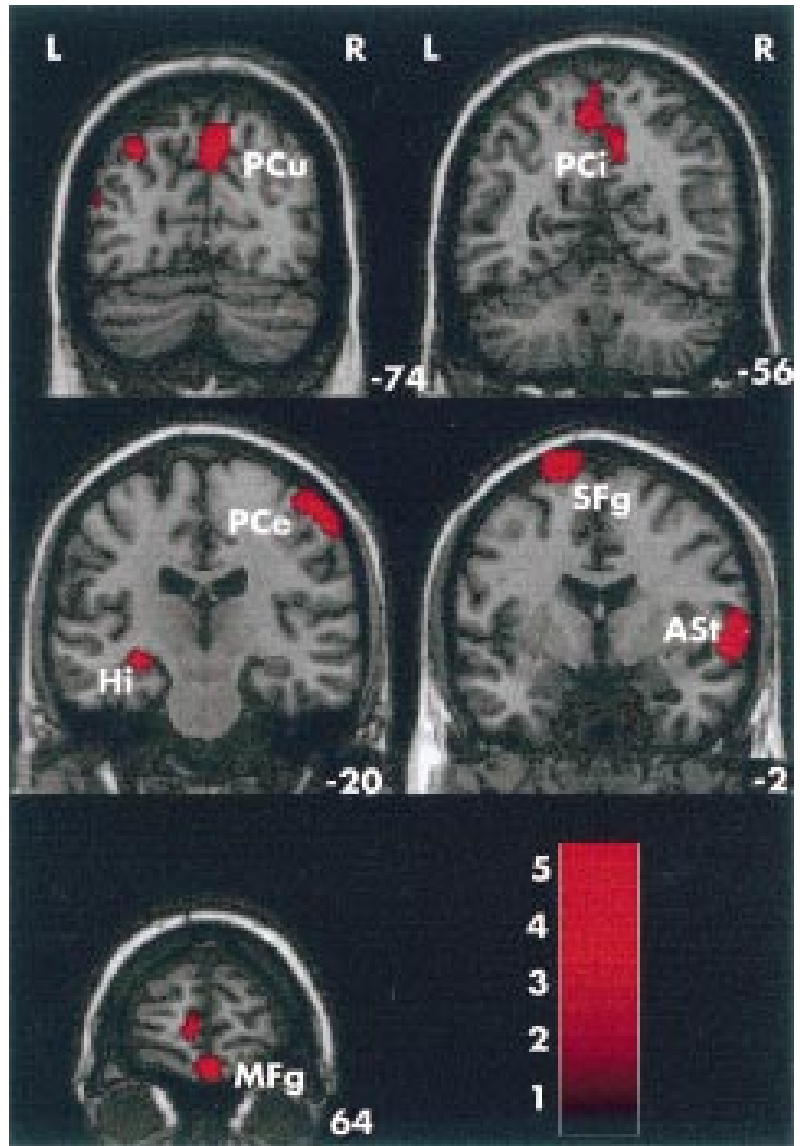

Figure 2 The images display brain regions where there were regional cerebral blood flow (rCBF) reductions in patients with Alzheimer's disease $(n=9)$ relative to healthy controls $(n=10)$ during the memory activation paradigm, at the initial cut off of $Z=2.33(p<0.01)$, overlaid on coronal sections from a reference brain spatially normalised according to the Talairach and Tornoux stereotactic atlas. ${ }^{37}$ The numbers at the right bottom corner of each coronal view provide the $y$ axis coordinate values. Labels have been placed close to those foci of $\mathrm{rCBF}$ difference that were located in regions where abnormalities had been predicted a priori in the Alzheimer's disease group, and which reached the one tailed $p<0.001$ level of statistical significance $(Z=3.09)$, uncorrected for multiple comparisons. These included: the right anterior superior temporal gyrus (ASt); the right and left precuneus (PCu) extending towards the posterior cingulate gyrus (PCi); the left hippocampus $(\mathrm{Hi})$; the right postcentral gyrus (PCe); the upper portion of the left superior frontal gyrus (SFg); and the anterior portion of the right medial frontal gyrus (MFg). None of those regions showed significantly decreased $\mathrm{rCBF}$ values in Alzheimer patients compared with controls when the SPECT scans obtained during the control condition were compared between the groups. L, left hemisphere; $R$, right hemisphere.

Alzheimer patients ( $p<0.001$, uncorrected) in two foci in the prefrontal cortex, involving, respectively, the upper portion of the right superior and middle frontal gyri (59l voxels; $\left.\mathrm{Z}_{\max }=3.42 ; \mathrm{x}, \mathrm{y}, \mathrm{z}=44,18,50 ; \mathrm{BA} 9 / 8\right)$, and the left lateral inferior frontal gyrus (96 voxels; $\mathrm{Z}_{\max }=3.44 ; \mathrm{x}, \mathrm{y}, \mathrm{z}=-54,38,4$; BA45/46). In addition, memory related rCBF increases were greater in the controls than in the Alzheimer patients in several temporal and parietal regions, including the left inferior parietal lobule and post-central gyrus $\left(\mathrm{Z}_{\max }=4.25\right.$; $\mathrm{x}, \mathrm{y}, \mathrm{z}=-52,-32,54 ; \mathrm{BA} 40 / 2)$; the posterior portions of the left middle $\left(\mathrm{Z}_{\max }=3.97 ; \mathrm{x}, \mathrm{y}, \mathrm{z}=-42,-66,14 ; \mathrm{BA19}\right)$ and inferior temporal gyri $\left(Z_{\max } 3.21 ; x, y, z=-56,-42,-18\right.$; BA37/20); the right anterior superior temporal gyrus $\left(Z_{\max } 3.47\right.$; $\mathrm{X}, \mathrm{y}, \mathrm{Z}=62,-2,0 ; \mathrm{BA22})$; and the right precuneus $\left(\mathrm{Z}_{\max }=3.39\right.$; $\mathrm{x}, \mathrm{y}, \mathrm{Z}=14,-48,48$; BA7). Conversely, memory related rCBF increases were greater in the Alzheimer patients than in the 
Table 2 Brain regions where regional cerebral blood flow values during the memory task were significantly correlated with measures of hippocampal grey matter atrophy in subjects with Alzheimer's disease $(n=9)$

\begin{tabular}{|c|c|c|c|c|c|}
\hline Areas of correlation & $x^{*}$ & $y^{*}$ & $\mathrm{z}^{*}$ & Zmax $†$ & Size $\neq$ \\
\hline \multicolumn{6}{|l|}{ Direct } \\
\hline R superior temporal gyrus (BA 22/38) & 66 & -8 & -34 & 4.24 & 907 \\
\hline L middle temporal gyrus (BA 21) & -66 & -14 & -26 & 4.04 & 398 \\
\hline R superior temporal gyrus (BA 22) & 64 & -16 & 0 & 3.94 & 420 \\
\hline L hippocampus/parahippocampal gyrus & -26 & -34 & -14 & 3.76 & 82 \\
\hline $\mathrm{L}$ posterior cingulate gyrus (BA 31 ) & -4 & -42 & 34 & 3.49 & 265 \\
\hline $\begin{array}{l}\text { R superior temporal gyrus (BA 22) } \\
\text { Inverse }\end{array}$ & \multicolumn{4}{|c|}{ Inverse } & 321 \\
\hline $\mathrm{R}$ inferior frontal gyrus/insula§ & 32 & -10 & 10 & 4.42 & 1152 \\
\hline $\mathrm{L}$ inferior frontal gyrus/insula $\S$ & -40 & 4 & 14 & 4.18 & 1179 \\
\hline $\mathrm{L}$ inferior frontal gyrus (BA 10/46) & -46 & 26 & 8 & 3.52 & 170 \\
\hline $\mathrm{R}$ inferior frontal gyrus (BA $10 / 46)$ & 40 & 26 & 14 & 3.27 & 166 \\
\hline$R$ and $L$ cerebella§ & 22 & -58 & -32 & 3.78 & 1821 \\
\hline
\end{tabular}

controls in a large bilateral inferior frontal cluster (with 3333 surpassing the initial threshold of $\mathrm{Z}=2.33)(\mathrm{p}<0.001$, corrected for multiple comparisons). This cluster had its voxel of peak statistical significance located in the inferolateral portion of the right middle frontal gyrus $\left(\mathrm{Z}_{\max }=4.08\right.$; $\mathrm{x}, \mathrm{y}, \mathrm{z}=30,50,2)$, and also included the right and left orbital and rectal gyri (BAll and BA47); the right and left subcallosal medial frontal cortex (BA25); and additional inferolateral portions of the right inferior, middle, and superior frontal gyri (BAl0/1l/47, up to the $\mathrm{z}$ coordinate level of +12 ). Memory related $\mathrm{rCBF}$ increases were also greater in Alzheimer patients than in the controls in a smaller cluster ( 158 voxels) located in the left dorsal medial prefrontal and anterior cingulate cortices $\left(Z_{\max }=3.47 ; \mathrm{x}, \mathrm{y}, \mathrm{Z}=-14,34,28 ; \mathrm{BA} 9 / 32\right)$.

\section{Correlations between medial temporal atrophy and rCBF patterns in the Alzheimer group}

Table 2 summarises the results of the voxel based correlational analysis calculated in the Alzheimer group between the $\mathrm{rCBF}$ measures obtained during the memory task and the measure of grey matter concentration in the hippocampal region, including the total amount of grey matter in the brain as a confounding covariate. Significant direct correlations ( $p<0.001$, uncorrected) were seen between the hippocampal grey matter concentration and rCBF values in two of the regions where regional hypoactivity had been detected in the Alzheimer patients relative to the controls-namely, the right anterior superior temporal gyrus (BA22/38) and the left hippocampus/parahippocampal gyrus (table 2). These results indicated that the rCBF values in those areas were lower in direct proportion to the degree of hippocampal atrophy in the Alzheimer group. In addition, significant correlations in the same direction were seen between the hippocampal grey matter concentration and rCBF values during the memory task in the right posterior superior temporal gyrus (BA22), the left middle temporal gyrus (BA21), and the left posterior cingulate cortex (BA31) (table 2). These correlations are illustrated in fig 3 (panels A-D). During the baseline condition, none of those regions showed significant rCBF correlations with the measures of hippocampal grey matter concentration, even on inspection using a less strict statistical cut off point $(\mathrm{p}<0.01$, uncorrected).

In contrast with the findings above, there were four voxel clusters in the frontal cortex where rCBF values during the memory task showed significant inverse correlations with the measures of hippocampal grey matter concentration (table 2).
Two of these clusters were located bilaterally in the inferior frontal gyrus/insula, extending on the left side to the precentral gyrus; both of these retained significance after correction for multiple comparisons $(p<0.05)$. These inverse correlations are illustrated as plots in fig 3 (panels E and F). The two other clusters were situated bilaterally in a more anterior and lateral portion of the inferior frontal gyrus (BA10/46) $(p<0.001$, uncorrected $)$. These results indicated that the greater the degree of hippocampal atrophy in the Alzheimer patients, the higher the rCBF values in those frontal areas during the memory task. Similar patterns were seen also during the baseline condition, with the degree of hippocampal atrophy showing significant inverse correlations with $\mathrm{rCBF}$ values in the left middle frontal gyrus (BA9; $\mathrm{Z}_{\max }=3.20$; $\mathrm{x}, \mathrm{y}, \mathrm{z}=-50,8,38 ; \mathrm{p}<0.001$ uncorrected), as well as in the inferior frontal gyrus/insula on both the right hemisphere $\left(\mathrm{Z}_{\max }=5.22 ; \mathrm{x}, \mathrm{y}, \mathrm{Z}=34,10,-4 ; \mathrm{p}<0.001\right.$ corrected for multiple comparisons $)$ and the left hemisphere $\left(Z_{\max }=5.14\right.$; $\mathrm{x}, \mathrm{y}, \mathrm{z}=-40,12,0 ; \mathrm{p}<0.001$ corrected). Finally, one unpredicted inverse correlation in the Alzheimer group was seen between the measure of hippocampal grey matter concentration and rCBF values during the memory task in a voxel cluster encompassing both cerebellar hemispheres, which retained statistical significance after correction for multiple comparisons $(\mathrm{p}<0.001)$ (table 2$)$.

\section{DISCUSSION}

In this study, we acquired both structural MRI and rCBF SPECT data in the same sample of subjects with mild Alzheimer's disease and healthy controls, in order to investigate the relation between hippocampal atrophy and rCBF patterns elicited during the performance of a verbal memory retrieval task.

Before calculating correlations between structural and functional imaging indices in Alzheimer patients, we did between-group comparisons on the data with both modes, and obtained results that are broadly in agreement with previous imaging studies in Alzheimer's disease. Thus we detected bilateral reductions in grey matter concentration in Alzheimer patients relative to controls in the hippocampal region, consistent both with the results of ROI based morphometric studies ${ }^{79}$ and with more recent studies using the VBM approach. ${ }^{43}{ }^{44}$ Also, the between-group SPECT comparisons showed significant rCBF reductions in Alzheimer patients relative to controls in the expected sites, including the lateral 
A

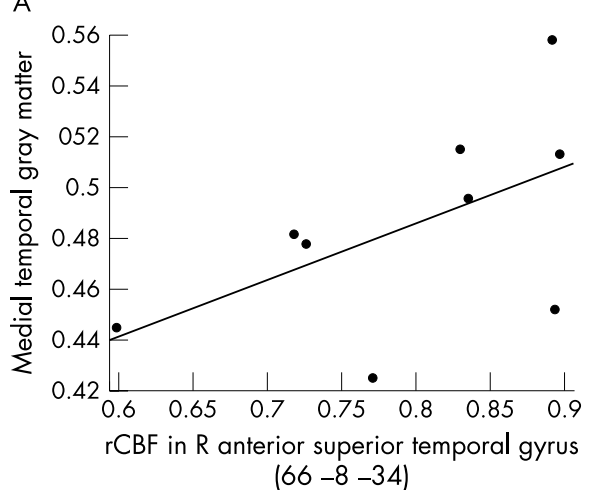

C

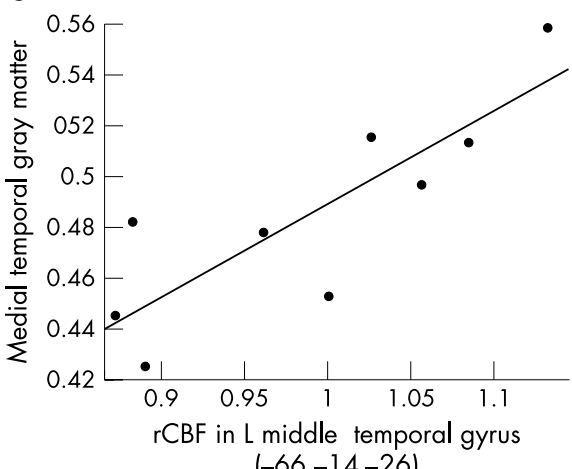

$(-66-14-26)$

E

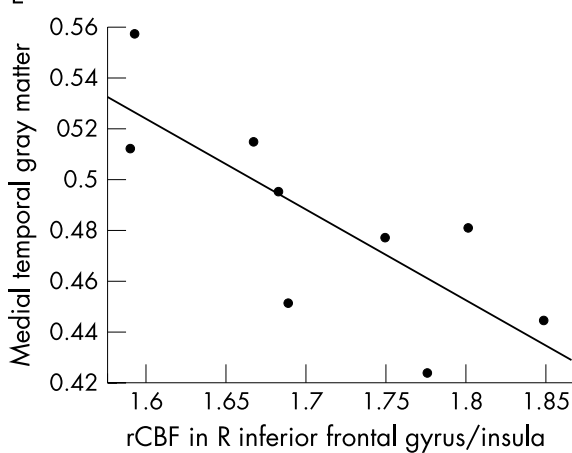

(32-10 10)
B

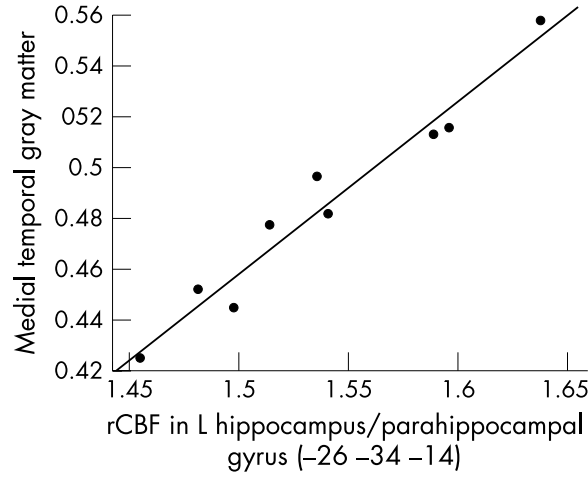

D

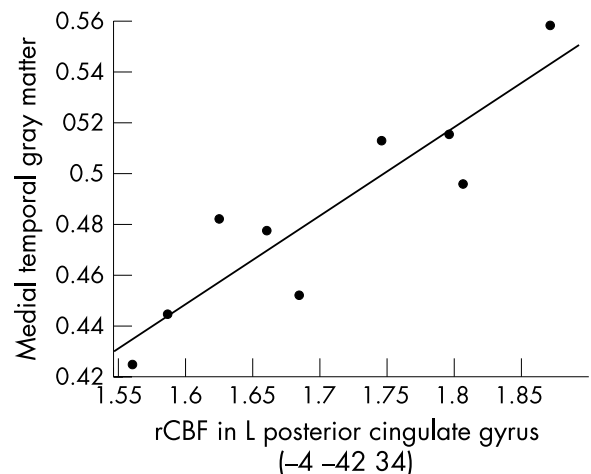

F

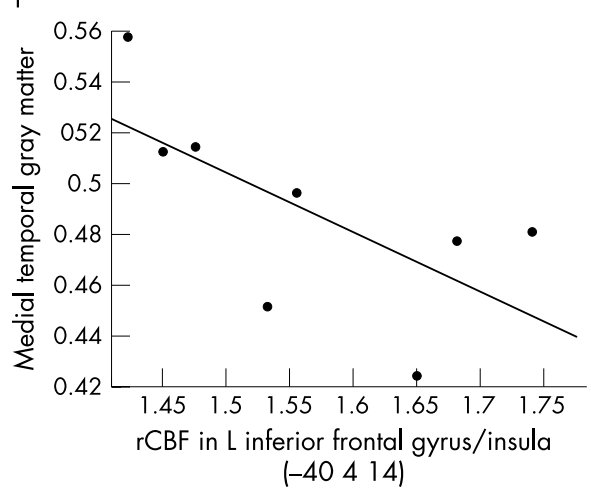

Figure 3 (A-D) Plots of the significant direct linear correlations $(p<0.001)$ observed in the Alzheimer's disease group $(n=9)$ between the mean grey matter concentration in the medial temporal region and regional cerebral blood flow (rCBF) measures obtained during the memory task in: (A) the right anterior superior temporal gyrus; (B) the left hippocampus/parahippocampal gyrus; (C) the left middle temporal gyrus (BA21); (D) the left posterior cingulate cortex (BA31). (E, F) Plots of the significant inverse correlations $(p<0.001)$ between the measure of hippocampal grey matter and $\mathrm{rCBF}$ in $(\mathrm{E})$ the right and $(\mathrm{F})$ left inferior frontal gyrus/insula. In all analyses, the total amount of grey matter in the brain was treated as a confounding covariate. The numbers between brackets in the $\mathrm{x}$ axes correspond to the coordinates of the voxel from which the $\mathrm{rCBF}$ indices were obtained. Further statistical details are provided in table 2. L, left hemisphere; R, right hemisphere.

and medial temporal cortices and the parietal lobe. Such rCBF reductions in Alzheimer patients were seen during the memory task but not during the control condition; this pattern is consistent with the findings of earlier PET and SPECT studies, ${ }^{18}{ }^{45}$ and supports the view that the performance of complex cognitive tasks may enhance regional brain metabolic differences between Alzheimer patients and healthy controls. ${ }^{45}$ Finally, our Alzheimer subjects showed significantly broader rCBF increases in the frontal cortex than the controls during the verbal memory task, although this involved more inferiorly located regions compared with the prefrontal activation foci seen in the healthy control group. These results are consistent with previous PET studies which have included the orbitofrontal cortex among the frontal cortical sites where compensatory rCBF increases may occur in Alzheimer patients during episodic memory activation paradigms. ${ }^{23}$
With regard to the relation between the structural MRI and SPECT data, we detected significant direct correlations between the degree of hippocampal atrophy in Alzheimer patients and rCBF reductions bilaterally in the temporal neocortex, as well as in the left hippocampus and the left posterior cingulate cortex. While the correlations involving the temporal neocortex are in agreement with the results of previous resting functional imaging studies of Alzheimer's disease, ${ }^{27-29}$ significant correlations between hippocampal atrophy and the functional activity in limbic regions have been absent in those studies. ${ }^{28}{ }^{29}$ It is possible that our correlations involving the temporo-limbic cortex and the posterior cingulate gyrus were detected because of the greater sensitivity of the voxel based methodology in comparison with the traditional ROI approach for the identification of findings in medially located structures of complex anatomy. However, in the study by 
Meguro et al $^{29}$ the resting glucose metabolic values in the entire brain were inspected using a similar voxel based approach, and significant correlations with the indices of hippocampal atrophy were restricted to lateral neocortical portions of the temporal and parietal cortices. It could be argued that our medial temporal functional-structural correlations were determined by partial volume effects caused by local atrophy on the rCBF measurements, as these were obtained in our study using a SPECT imaging technique with limited spatial resolution. ${ }^{46}$ However, an influence of artefactual partial volume effects on our correlational results is unlikely, as our significant direct relation between hippocampal atrophy and $\mathrm{rCBF}$ values in the left hippocampus emerged only during the verbal retrieval task and not during the control condition; the same applied for the correlation between hippocampal atrophy and $\mathrm{rCBF}$ values in the left posterior cingulate cortex. This indicates that, rather than being static, the relation between hippocampal atrophy and functional activity in limbic regions in our study was modulated by the cognitive state during which the rCBF tracer ${ }^{99 m}$ Tc-HMPAO was given.

It is interesting that the posterior cingulate gyrus (BA 31) was the only region beyond the temporal cortex that showed significant direct rCBF correlations with the degree of hippocampal atrophy in our Alzheimer patients during the memory task. The posterior cingulate cortex, affected early in Alzheimer's disease and in subjects at genetic risk for the disorder, ${ }^{47}$ has reciprocal anatomical connections with the parahippocampal cortex ${ }^{48}$ and has also been implicated in the functional imaging literature of memory activation in healthy subjects. ${ }^{25}$ In a voxel based PET study of resting glucose metabolism in Alzheimer's disease, regional metabolic deficits in the left posterior cingulate cortex and the left hippocampal/ parahippocampal region were specifically related to impairments in verbal episodic memory, while the performance in other memory domains correlated significantly with resting metabolic deficits in different brain regions. ${ }^{49}$ Together with those findings, our correlational results suggest that the degree of hippocampal atrophy influences the disrupted functioning of a left sided network that is particularly relevant to verbal episodic memory deficits in Alzheimer's disease, involving the medial temporal cortex and the interconnected posterior cingulate gyrus.

In contrast to the above findings, we detected frontal cortical clusters where rCBF values during the memory task were inversely correlated with the hippocampal grey matter concentration in the Alzheimer group. These findings are consistent with the view that frontal rCBF increases in Alzheimer patients studied during episodic retrieval paradigms are compensatory responses, related to the increased effort needed to perform the memory tasks. ${ }^{192326}$ The correlational patterns reported here suggest that compensatory changes in activity in the frontal cortex may be elicited in Alzheimer patients in direct proportion to the degree of anatomical abnormality in the hippocampal areas primarily affected by the neuropathological disease process. However, caution is needed with this interpretation as there was no spatial overlap between the frontal clusters, where rCBF correlated significantly with the MRI measures, and the orbitofrontal areas, where rCBF increases were seen during the memory task relative to the control condition in the Alzheimer group. On the other hand, this apparent inconsistency could be explained by the fact that the significant correlations between hippocampal grey matter and $\mathrm{rCBF}$ values in the inferior frontal gyrus/insula were present not only during the memory task but also during the control condition. This pattern of results indicates that the degree of hippocampal neuronal damage in Alzheimer's disease may influence the emergence of compensatory frontal rCBF increases not only during memory processes but also during other cognitive operations of lesser complexity.

\section{Conclusions and limitations}

Our results provide an indication that hippocampal atrophic changes in Alzheimer's disease are associated with reduced functioning of temporal and posterior cingulate regions during verbal episodic retrieval, but with increased activity in frontal areas, probably on a compensatory basis. However, there are important limitations to the study which should also be highlighted.

First, the relatively long duration of the SPECT imaging session and the need to perform MRI measurements on a separate occasion complicated our recruitment of a larger study sample. Although the inspection of our data did not indicate the influence of outliers on the correlational patterns detected, replication of the present findings with larger groups is clearly needed.

Second, our Alzheimer sample included subjects who were receiving stable doses of psychotropic drugs that may have influenced our rCBF measurements. In particular, it has been suggested that cognition enhancing cholinergic agents reduce task related $\mathrm{rCBF}$ activation in the frontal cortex in healthy subjects, ${ }^{50}$ as well as decreasing the rate of resting brain functional decline in Alzheimer patients. ${ }^{51}{ }^{52}$ It is thus possible that the use of those drugs in a proportion of our Alzheimer subjects might have prevented us from demonstrating larger between-group rCBF differences or greater significant correlations between hippocampal atrophy and hypofunctional patterns in the Alzheimer group.

Finally, the limited temporal resolution of the SPECT methodology and the restriction of performing only two acquisitions in the same session prevented us from separately assessing the rCBF patterns elicited by each of the several cognitive processes involved in the verbal recognition memory task. Such limitations could be overcome in imaging studies using novel paradigms combining structural and functional MRI (fMRI). Using event related designs with memory tasks, the fMRI technique may successfully detect haemodynamic changes associated with single trials of item recognition and rejection, as well as discriminating those transient functional responses from the activity patterns associated with the maintenance of the retrieval mode..$^{53-55}$ Future studies using those paradigms may be ideally suited to extending the preliminary correlational results reported here.

\section{ACKNOWLEDGEMENTS}

This work was supported by Fundação de Amparo à Pesquisa do Estado de São Paulo (FAPESP), Brazil, projects 99/09547-3, 97/ 14206-5, 95/9446-1 and 99/12205-7.

\section{Authors' affiliations}

G E J Garrido, S S Furuie, Division of Informatics, Heart Institute (InCor), University of São Paulo Medical School, Brazil

C A Buchpiguel, C C Castro, Department of Radiology, Faculty of Medicine, University of São Paulo

C M C Bottino, C G Cid, C H P Camargo, G F Busatto, Department of Psychiatry, Faculty of Medicine, University of São Paulo

O P Almeida, Department of Psychiatry and Behavioural Science, University of Western Australia, Australia

M F Glabus, Unit of Integrative Neuroimaging, Clinical Brain Disorders Branch, National Institutes of Health, USA

Competing interests: none declared.

\section{REFERENCES}

1 Terry RD, Katzman R. Senile dementia of the Alzheimer type. Ann Neurol 1983;14:497-506.

2 Braak H, Braak E. Neuropathological staging of Alzheimer-related changes. Acta Neuropathol (Berl) 1991;82:239-59.

3 Arriagada PV, Growdon JH, Hedley-Whyte ET, et al. Neurofibrillary tangles but not senile plaques parallel duration and severity of Alzheimer's disease. Neurology 1992;42:631-9.

4 Squire LR, Zola-Morgan S. The medial temporal lobe memory system. Science 1991;253:1380-6.

5 O'Brien JT. Is hippocampal atrophy on magnetic resonance imaging a marker for Alzheimer's disease? Int J Geriatr Psychiatry 1995;10:43 1-5. 
6 Laakso MP, Soininen H, Partanen K, et al. Volumes of hippocampus, amygdala and frontal lobes in the MRI-based diagnosis of early Alzheimer's disease: correlation with memory functions. J Neural Transm Park Dis Dement Sect 1995;9:73-86.

7 Jack CR, Petersen RC, Xu YC, et al. Medial temporal atrophy on MRI in normal aging and very mild Alzheimer's disease. Neurology 1997:49:786-94

8 Pantel J, Schroder J, Schad LR, et al. Quantitative magnetic resonance imaging and neuropsychological functions in dementia of the Alzheimer type. Psychol Med 1997;27:221-9.

9 Krasuski JS, Alexander GE, Horwitz B, et al. Volumes of medial temporal lobe structures in patients with Alzheimer's disease and mild cognitive impairment (and in healthy controls). Biol Psychiatry 1998;43:60-8.

10 Petersen RC, Jack CR, Xu YC, et al. Memory and MRI-based hippocampal volumes in aging and AD. Neurology 2000;54:581-7.

11 Gemmell HG, Sharp PF, Besson JA, et al. Differential diagnosis in dementia using the cerebral blood flow agent $99 \mathrm{mT}$ C HM-PAO: a SPECT study. J Comput Assist Tomogr 1987; 1 1:398-402.

12 Costa DC, Ell PJ, Burns A, et al. CBF tomograms with [99mTc]-HM-PAO in patients with dementia (Alzheimer type and HIV) and Parkinson's disease - initial results. J Cereb Blood Flow Metab 1988;8:S109-15.

13 Rapoport SI, Horwitz B, Grady CL, et al. Abnormal brain glucose metabolism in Alzheimer's disease, as measured by position emission tomography. Adv Exp Med Biol 1991;291:231-48.

14 Holman BL, Johnson KA, Gerada B, et al. The scintigraphic appearance of Alzheimer's disease: a prospective study using technetium-99m-HMPAO SPECT. J Nucl Med 1992;33:181-5.

15 Ohnishi T, Hoshi H, Nagamachi S, et al. High-resolution SPECT to assess hippocampal perfusion in neuropsychiatric diseases. J Nucl Med 1995;36:1163-9.

16 Kogure D, Matsuda H, Ohnishi T, et al. Longitudinal evaluation of early Alzheimer's disease using brain perfusion SPECT. J Nucl Med 2000;41:1155-62.

17 Matsuda $\mathbf{H}$. Cerebral blood flow and metabolic abnormalities in Alzheimer's disease. Ann Nucl Med 2001;15:85-92.

18 Riddle W, O'Carroll RE, Dougall N, et al. A single photon emission computerised tomography study of regional brain function underlying verbal memory in patients with Alzheimer-type dementia. Br J Psychiatry 1993;163:166-72.

19 Becker JT, Mintun MA, Aleva K, et al. Compensatory reallocation of brain resources supporting verbal episodic memory in Alzheimer's disease. Neurology 1996;46:692-700.

20 Woodard JL, Grafton ST, Votaw JR, et al. Compensatory recruitment of neural resources during overt rehearsal of word lists in Alzheimer's disease. Neuropsychology 1998;12:491-504.

21 Cardebat D, Demonet JF, Puel M, et al. Brain correlates of memory processes in patients with dementia of Alzheimer's type: a SPECT activation study. J Cereb Blood Flow Metab 1998;18:457-62

22 Saykin AJ, Flashman LA, Frutiger SA, et al. Neuroanatomic substrates of semantic memory impairment in Alzheimer's disease: patterns of functional MRI activation. J Int Neuropsychol Soc 1999;5:377-92.

23 Backman L, Andersson JL, Nyberg L, et al. Brain regions associated with episodic retrieval in normal aging and Alzheimer's disease. Neurology 1999.52:1861-70.

24 Grady CL, Furey ML, Pietrini P, et al. Altered brain functional connectivity and impaired short-term memory in Alzheimer's disease. Brain 2001; 124:739-56.

25 Cabeza R, Nyberg L. Imaging cognition II: an empirical review of 275 PET and fMRI studies. J Cogn Neurosci 2000;12:1-47.

26 Rapoport SI. In vivo PET imaging and postmortem studies suggest potentially reversible and irreversible stages of brain metabolic failure in Alzheimer's disease. Eur Arch Psychiatry Clin Neurosc 1999:249:46-55.

27 Jobst KA, Smith AD, Barker CS, et al. Association of atrophy of the medial temporal lobe with reduced blood flow in the posterior parietotemporal cortex in patients with a clinical and pathological diagnosis of Alzheimer's disease. J Neurol Neurosurg Psychiatry 1992:55:190-4.

28 Yamaguchi S, Meguro K, Itoh M, et al. Decreased cortical glucose metabolism correlates with hippocampal atrophy in Alzheimer's disease as shown by MRI and PET. J Neurol Neurosurg Psychiatry 1997;62:596-600

29 Meguro K, LeMestric C, Landeau B, et al. Relations between hypometabolism in the posterior association neocortex and hippocampa atrophy in Alzheimer's disease: a PET/MRI correlative study. J Neurol Neurosurg Psychiatry 2001;71:315-21.

30 McKhann G, Drachman D, Folstein M, et al. Clinical diagnosis of Alzheimer's disease: report of NINCDS/ADRDA Work Group under the auspices of Department of Health and Human Services Task Force on Alzheimer's disease. Neurology 1984;34:939-44

31 Roth M, Tym E, Mountjoy CQ, et al. CAMDEX. A standardised instrument for the diagnosis of mental disorder in the elderly with special reference to the early detection of dementia. Br J Psychiatry 1986:149:698-709.

32 Folstein MF, Folstein SE, McHugh PR. Mini mental state: a practical method for grading the cognitive state of patients for the clinician. J Psychiatr Res 1975;12:189-98.

33 Associação Brasileira de Anunciantes. XXXI Estudos Marplan. São Paulo: Marplan Brasil Representações e Pesquisas Ltda, 1989.

34 Monsch AU, Bondi MW, Butters $N$, et al. Comparisons of verbal fluency tasks in the detection of dementia of the Alzheimer type. Arch Neurol 1992; 49: 1253-8.

35 Fuld PA, Masur DM, Blau AD, et al. Object-memory evaluation for prospective detection of dementia in normal functioning elderly: predictive and normative data. J Clin Exp Neuropsychol 1990; 12:520-8

36 Ashburner J, Friston KJ. Voxel-based morphometry - the methods Neuroimage 2000;11:805-21.

37 Talairach J, Tornoux P. Co-planar stereotaxic atlas of the human brain. New York: Thieme, 1988.

38 Busatto GF, Costa DC, Ell PJ, et al. Regional cerebral blood flow (rCBF) in schizophrenia during verbal memory activation: a $99 \mathrm{mTc}-\mathrm{HMPAO}$ single photon emission tomography (SPET) study. Psychol Med 1994; 24:463-72.

39 Andersen AR, Friberg HH, Schmidt JF, et al. Quantitative measurements

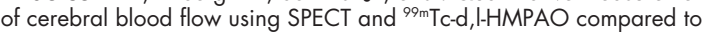
xenon-133. J Cereb Blood Flow Metab 1988;8:S69-81.

40 Friston KJ, Holmes AP, Worsley K, et al. Statistical parametric maps in functional imaging: a general linear approach. Hum Brain Mapping 1995; 2:189-210.

41 Friston KJ, Holmes A, Poline JB, et al. Detecting activations in PET and fMRI: levels of inference and power. Neuroimage 1996;4:223-35.

42 Busatto GF, Garrido GE, Almeida OP, et al. A voxel-based morphometry study of temporal lobe grey matter reductions in Alzheimer's disease. Neurobiol Aging 2002 (in press).

43 Rombouts SA, Barkhof F, Witter MP, et al. Unbiased whole-brain analysis of gray matter loss in Alzheimer's disease. Neurosci Lett 2000;285:231-3.

44 Baron JC, Chetelat G, Desgranges B, et al. In vivo mapping of gray matter loss with voxel-based morphometry in mild Alzheimer's disease. Neuroimage 2001;14:298-309.

45 Kessler J, Herholz K, Grond M, et al. Impaired metabolic activation in Alzheimer's disease: a PET study during continuous visual recognition. Neuropsychologia 1991;29:229-43

46 Meltzer CC, Zubieta JK, Brandt J, et al. Regional hypometabolism in Alzheimer's disease as measured by positron emission tomography after correction for effects of partial volume averaging. Neurology 1996:47:454-61.

47 Small GW, Ercoli LM, Silverman DH, et al. Cerebral metabolic and cognitive decline in persons at genetic risk for Alzheimer's disease. Proc Natl Acad Sci USA 2000;97:6037-42.

48 Pandya DN, Van Hoesen GW, Mesulam MM. Efferent connections of the cingulate gyrus in the rhesus monkey. Exp Brain Res $1981 ; 42: 319-30$

49 Desgranges B, Baron JC, de la Sayette V, et al. The neural substrates of memory systems impairment in Alzheimer's disease. A PET study of resting brain glucose utilization. Brain 1998:121:611-31.

50 Furey ML, Pietrini P, Haxby JV, et al. Cholinergic stimulation alters performance and task-specific regional cerebral blood flow during working memory. Proc Natl Acad Sci USA 1997;94:6512-6.

51 Nakano S, Asada T, Matsuda H, et al. Donepezil hydrochloride preserves regional cerebral blood flow in patients with Alzheimer's disease. J Nucl Med 2001:42:1441-5.

52 Potkin SG, Anand R, Fleming K, et al. Brain metabolic and clinical effects of rivastigmine in Alzheimer's disease. Int J Neuropsychopharmacol 2001;4:223-30.

53 McDermott KB, Jones TC, Petersen SE, et al. Retrieval success is accompanied by enhanced activation in anterior prefrontal cortex during recognition memory: an event-related fMRI study. J Cogn Neurosci 2000; 12:965-76.

54 Donaldson DI, Petersen SE, Ollinger JM, et al. Dissociating state and item components of recognition memory using fMRI. Neuroimage $2001 ; 13: 129-42$

55 Daselaar SM, Rombouts SA, Veltman DJ, et al. Parahippocampal activation during successful recognition of words: a self-paced event-related fMRI study. Neuroimage 2001;13:1113-20.

56 Montgomery AS, Åsberg M. A new depression scale designed to be sensitive to change. Br J Psychiatry 1979;134:382-9. 